\title{
Optimal Ordering Policy of Deteriorating Items with Mixed Cargo Transportation over a Finite Planning Horizon
}

\author{
Hsi-Mei Hsu, Zi-Yin Chen \\ Department of Industrial Engineering \& Management, National Chaio Tung University, \\ Hsinchu, Chinese Taipei \\ Email: hsimei@cc.nctu.edu.tw, yahuilin2@ydu.edu.tw
}

Received January 21, 2012; revised February 27, 2012; accepted March 8, 2012

\begin{abstract}
In this paper, we propose a deteriorating items inventory model with constant demand and deterioration rates, and mixed cargo transportation modes. The transportation modes are full container load (FCL) and less than container load (LCL). Deteriorating items, such as specialty gases which are applied in semiconductor fabrication, deteriorate owing to environmental variation. Exact algorithms are proposed to determine the optimal inventory policies over a finite and an infinite planning horizon. Numerical examples are given to illustrate the proposed solution procedures. In addition, when the deterioration rate is large, the results of the proposed model perform better compared to the inventory model proposed by Rieksts and Ventura (2008).
\end{abstract}

Keywords: Transportation Costs; Deteriorating Items; Inventory Management

\section{Introduction}

In this paper, we study a deteriorating items inventory model with mixed cargo transportation (full container load, FCL, and less than container load, LCL) over a given finite planning horizon. The customer's demand per unit time $d$ is constant and shortage is not allowed. The retailer places orders to its supplier and goods are transported by cargos. FCL and LCL cargoes are used. LCL is a shipment of cargo by which goods do not fully fill the container. The remaining space of the Less than Container may be filled with goods of other shippers. Generally, the transportation cost per unit under FCL is cheaper than LCL. Therefore, if goods can fill an entire container, FCL cargo will be the first choice.

In this paper, the assumptions are as follows: 1 ) supplier capacity is unlimited; 2) the delivery time is constant; 3) the salvage of inventory is zero; 4) the retailer needs to satisfy customers' demand and shortage is not allowed. The retailer intends to determine the optimal order interval in order to minimize the total cost.

In Taiwan's semiconductor fabrication industries, many manufacturers need to import raw materials from abroad, such as certain specialty gases which may deteriorate owing to environmental variation. This research is motivated by a Taiwanese gas importing company. The gas importing company makes gas supply contracts with customers for a certain time period, and the contracts indicate that the retailers shall supply raw materials to meet customers' demands within a given time period. FCL and LCL are allowed. For example, Company A is the top supplier of electronic specialty gases in Taiwan, and its' customers include many international semiconductor manufacturing companies. Company A also provides the on-site gas generation system that includes small membrane cabinets, packaged plants, large air separation and hydrogen/carbon monoxide plants and industrial gas pipelines. Generated Gas Systems benefit customers by not only providing economical gas supply but also creating environmental advantages through reduced truck deliveries. In the gas generation system, since gas leakage cannot be completely prevented or avoided owing to miss operation or gas pipeline deterioration and failure, the gas monitoring system is built to detect the amount of gas leakage. In addition, Company A has signed supply contracts with various semiconductor manufacturing companies to supply various types of bulk gas, bulk specialty gas, and electronic chemicals. Under these contracts, Company A is obliged to supply gas to meet customers' demand ( $\left.d T_{H}\right)$ within a finite horizon $T_{H}$ and shortage is not allowed. However, Company A needs to order gas from foreign suppliers in order to satisfy customers' demand for bulk gas, bulk specialty gas, and electronic chemicals. In addition to a fixed or- 
dering cost $(K)$, each order payment also includes fees related to the quantity of transportation containers and the number of items of LCL shipping.

After the termination of the contract, the surplus inventory still belongs to Company A and customers only need to pay for the quantity which they have used. Since surplus inventory of industrial gas in the on-site gas generation system may be disposed with additional costs, Company A therefore expects to reserve no surplus product after the termination of the contract. According to the above-mentioned situation, Company A should construct its own inventory model so as to determine the optimal order interval.

In this paper, we refer to Company A as retailer. We develop a deteriorating items inventory model with mixed cargo transportation to determine the order interval of goods over a given finite planning horizon. The model is formulated to minimize the retailer's expected cost. The following costs are considered. 1) The deteriorating costs of gas $C_{d}$ : the percentage of gas which has deteriorated per unit time is $\theta$. The purchase cost per unit of gas multiplying by the deterioration rate $\theta$ equals to $C_{d}$;2) Holding cost $h$ per unit time: the holding cost per unit is the sum of interest, insurance and the holding cost; 3) Setup cost $K$ per order; 4) Transportation cost: two kinds of transportation costs are considered. The first one is FCL cost which is calculated based on the number of containers used multiplying by the container fee per unit $C_{F}$. The other one is LCL cost.

Then we define the conditions for the existence of the optimal solution and develop a procedure to determine the optimal order interval. The rest of this paper is organized as follows: The assumptions and notations for the proposed model, the optimal ordering model and the solution procedure are proposed in Section 3. Some numerical examples are performed in Section 4. Finally, conclusions and future study are stated in Section 5.

\section{Literature Review}

In this paper, we propose the deteriorating items inventory model to determine the optimal order interval over the finite planning horizon. The retailer can receive goods with mixed cargo transportation (FCL and LCL). Nowadays mixed cargo is commonly used in international air or sea cargo shipping industry. The deteriorateing items inventory model with mixed cargo transportation (FCL and LCL) in this paper fall into the categories: 1) continuous review inventory problem with bi-modal transportation cost (Rieksts and Ventura [1]); 2) deteriorating items inventory model with deterministic demand and fixed lifetime (Goyal \& Giri [2], Li et al. [3]). Bimodal transportation problem discussed by Rieksts and Ventura [1] is truckload (TL) transportation and less than truckload (LTL). In fact, the inventory problem of the mixed cargo and bi-modal transportation cost are identical except that vehicles used. In TL and LTL, the vehicle is a truck but the vehicle becomes a container in our study. Thus, this paper reviewed recent studies of 1) continuous review inventory problem with bi-modal transportation cost; and 2) deteriorating items inventory model with deterministic demand and fixed lifetime.

Rieksts and Ventura [4] classified continuous review inventory models with bi-modal transportation cost as four types. The first type is TL with no freight discount. TL with no freight discount is that the cost per load does not change with the number of truckloads (Aucamp [5] and Lippman [6]). The second type is TL with quantity or freight discount, TL with quantity or freight discount is TL shipments with discounts on the cost per load or quantity as the number of truckloads increase (Lee [7], Hwang et al. [8], Tersine et al. [9]). The third type is TL and LTL with no freight discount. TL and LTL inventory models are discussed and compared simultaneously in this category (Adelwahab and Sargious [10]), Swenseth and Godfrey [11], Rieksts and Ventura [1]). The Fourth type is TL with other delivery. TL with other delivery is TL and LTL inventory models with the other options of delivery (e.g. shipping to each retailer directly or peddling deliveries to a customer (Burns et al. [12]), the shipment from origin, in-transit to the destination (Larson [13])).

Two extensive survey papers on deteriorating items inventory models have been published by Goyal \& Giri [2] and Li et al. [3]. Studies of deteriorating items inventory model with deterministic demand fixed lifetime are discussed respectively as follows; 1 ) deteriorating items inventory model with deterministic demand: Li et al. [3] classified deteriorating items inventory model with deterministic demand as five types of demand: fixed demand rate (Chung and Lin [14], Benkherouf et al. [15]), uniform demand (Heng et al. [16], Raafft et al. [17], Goh et al. [18]), time-varing demand (Yan and Cheng [19], Giri et al. [20], Teng et al. [21]), stock-dependent demand (Chung et al. [22], Giri and Chaudhuri [23], Bhattachaya [24], Wu et al. [25]) and price-depend demand (Wee [26], Wee and Law [27]); 2) deteriorating items inventory model with fixed lifetime: Li et al. [3] classified deteriorating items inventory model with fixed lifetime as two deteriorating rates: a constant deteriorating rate (Ghare and Schrader [28], Shah and Jaiswal [29], Padmanabhana and Vratb [30], Bhunia and Maiti [31]) and time-varing deteriorating rate (Bhunia and Maiti [32], Abad [33], Mukhopadhyay et al. [34], Mahapatra [35]).

The inventory models with a bi-modal transportation cost proposed by Rieksts and Ventura [1] is closely related to our problem. In this paper, we develop a more general deteriorating items inventory model with mixed 
cargo transportation to determine the optimal order interval, and the model of Rieksts and Ventura [1] is a subset of the proposed model. Under proposed model, the two types of transportation costs and the deteriorating cost of goods are formulated respectively in the deteriorating items inventory model to decide the optimal order interval.

\section{Problem Formulation}

\subsection{Notations}

The related notations are given as follows:

$\lceil x\rceil$ : integer operator, integer value equal or greater than $x$.

$\lfloor x\rfloor$ : integer operator, integer value equal or less than $x$.

$\theta$ : the constant deterioration rate of a unit gas volume per unit time, where $0<\theta \leq 1$.

$C_{d}$ : the unit deteriorated cost per unit gas volume per unit time, $C_{d}>0$.

$I(t)$ : on hand inventory at time $t$.

$d$ : demand rate is constant and known.

$h$ : holding cost per unit item per unit time.

$T_{H}$ : period of the whole planning horizon.

$n$ : order times during the entire period $T_{H}$.

$K$ : set up cost per order; $K>0$.

$C_{F}:$ the unit container cost.

$s$ : shipping cost of LTL per unit item.

$Q$ : the order quantity per cycle.

$Q_{C}$ : container maximum capacity.

$C(T)$ : the total transportation cost per cycle.

$T_{Q c}$ : the consumption duration for $Q_{C}$ that includes real demand and deteriorated items,

$$
T_{Q c}=\ln \left[(\theta / d) \cdot Q_{T}+1\right] / \theta .
$$

Decision variables:

$T$ : order interval. $T \geq 0$

Intermediate variables:

$\Omega_{1}$ : the space defined by

$$
\begin{aligned}
\Omega_{1}= & \left\{T \| T / T_{Q c}\right\rfloor \cdot T_{Q c} \leq T \leq\left\lfloor T / T_{Q c}\right\rfloor \cdot T_{Q c} \\
& \left.+\ln \left[(\theta / d) \cdot\left(C_{F} / s\right)+1\right] / \theta\right\}
\end{aligned}
$$

$\Omega_{2}$ : the decision space defined by

$$
\begin{aligned}
\Omega_{2} & =\left\{T \| T / T_{Q}\right\rfloor \cdot T_{Q}+\ln \left[(\theta / d) \cdot\left(C_{F} / s\right)+1\right] / \theta \\
& \left.<T<\left(\left\lfloor T / T_{Q}\right\rfloor+1\right) \cdot Q_{T}\right\}
\end{aligned}
$$

$T_{1}^{*}$ : optimal order interval in $\Omega_{1}$ over infinite planning horizon.

$T_{2}^{*}$ : optimal order interval in $\Omega_{2}$ over infinite planning horizon.

\subsection{Problem Assumptions and Formulation}

\subsubsection{Infinite Planning Horizon Model}

The mathematical model is formulated to determine the order interval with mixed cargo transportation for minimizing retailer's total cost. The customer's demand is a constant rate of $d$ units per unit time. The inventory deterioration rate $\theta$ is the percentage of a unit gas volume deteriorated per unit time. Therefore the inventory level will decrease owing to customer's demand and gas deterioration. Thus, the differential equation representing the inventory status (Ghare and Schrader [28]) is given by:

$$
\mathrm{d} I(t) / \mathrm{d} t+\theta I(t)+d=0, \quad 0 \leq t \leq T
$$

with the boundary condition $I(0)=Q$ and $I(T)=0$. The solution of (1) is:

$$
I(t)=(Q+d / \theta) \exp (-\theta t)-d / \theta, \quad 0 \leq t \leq T
$$

The order quantity per cycle can be written as:

$$
Q=(d / \theta)[\exp (\theta T)-1]
$$

where $T$ is the order interval. For ignorance of lead time and impermissible shortage, the optimal ordering policy is to place order when the inventory level is zero (zero inventory policy). Under the zero inventory policy to determine order quantity $Q$ is equivalent to determine the order interval $T$, i.e., order quantity $Q$ is equal to $d \cdot T$ plus total deteriorating quantities per cycle. Now we will formulate the retailer's total cost function per cycle, denoted as $T C(T)$, and determine the optimal order interval $T^{*}$ for infinite planning horizon.

The items considered in $T C(T)$ are total holding costs, total deteriorating costs, total set up costs and total transportation costs. The total cost function per unit time, denoted as $P T C(T)$, is given as follows:

$$
\begin{aligned}
T C(T)= & \text { Total holding costs }+ \text { Total deteriorating costs } \\
& + \text { Total set up costs }+ \text { Total transporation costs }
\end{aligned}
$$

$$
\begin{aligned}
\operatorname{PTC}(T)= & (\text { Total holding costs } \\
& + \text { Total deteriorating costs }+ \text { Total set up costs } \\
& + \text { Total transporation costs }) / T
\end{aligned}
$$

The relevant items in (4) are formulated respectively as follows:

1) Total holding cost per cycle

$$
=\int_{0}^{T} h \times I(t) \mathrm{d} x=\left(\mathrm{d} \times h / \theta^{2}\right)[\exp (\theta T)-\theta T-1]
$$

2) Total deteriorating costs per cycle: total deteriorateing quantities per cycle is $(Q-d T)$ and total deteriorated costs is $(Q-d T) \times C_{d}$. By (3), we get 


$$
\begin{aligned}
& (Q-d T) \times C_{d}=\{(d / \theta)[\exp (\theta T)-1]-d T\} \times C_{d} \\
& C(T)=\min \left\{\left[\left\lfloor T / T_{Q c}\right\rfloor C_{F}+s \cdot(d / \theta) \cdot\left\{\left[\exp \left[\theta \cdot\left(T-\left\lfloor T / T_{Q c}\right\rfloor \cdot T_{Q c}\right)\right]-1\right]\right\},\left(\left\lfloor T / T_{Q c}\right\rfloor+1\right) C_{F}\right\}\right.
\end{aligned}
$$

Because the unit item transportation cost with FCL is cheaper than it with LCL transportation, therefore FCL cargo is used first when order quantity $Q$ is larger than a container maximum capacity $Q_{C}$. The remaining goods are defined as $Q-\left\lfloor Q / Q_{C}\right\rfloor \cdot Q_{C}$ that will be transported by LCL or FCL. Goods transportation cost with FCL is charged by the amount of a container fee multiplied by total numbers of container used. Goods transportation cost with LCL is charged by unit transportation fee $s$ multiplied by remaining quantities. We assume $s Q_{C}>$ $C_{F}$.

Now we will discuss the relationships between $T$ and $T_{Q c}$. $T_{Q c}$ is the consumption length for quantity $Q_{C}$ that includes real demand plus deteriorated items. The relationship between $Q_{C}$ and $T_{Q c}$ is defined as follows by (3).

$$
Q_{C}=(d / \theta)\left[\exp \left(\theta T_{Q}\right)-1\right]
$$

Then $T_{Q}$ is:

$$
T_{Q c}=\ln \left[(\theta / d) \cdot Q_{C}+1\right] / \theta
$$

The numbers of FCL cargo used per cycle is between $\left\lfloor Q / Q_{C}\right\rfloor$ and $\left(\left\lfloor Q / Q_{C}\right\rfloor+1\right)$, i.e.,

$$
\left\lfloor Q / Q_{C}\right\rfloor \cdot Q_{C} \leq Q \leq\left(\left\lfloor Q / Q_{C}\right\rfloor+1\right) \cdot Q_{C} .
$$

In other words, the order interval $T$ is between $\left\lfloor T / T_{Q c}\right\rfloor$ and $\left(\left\lfloor T / T_{Q c}\right\rfloor+1\right)$, i.e.,

$$
\left\lfloor T / T_{Q c}\right\rfloor \cdot T_{Q c} \leq T \leq\left(\left\lfloor T / T_{Q c}\right\rfloor+1\right) \cdot T_{Q c}
$$

If $Q / Q_{C}$ is not an integer, then we must consider whether the remainder will be transported by FCL or LCL. The remaining quantities is equal to $\left(Q-\left\lfloor Q / Q_{C}\right\rfloor \cdot Q_{C}\right)$ that will be transported with LCL when $C_{F}$ is less than or equal to $s \cdot\left(Q-\left\lfloor Q / Q_{C}\right\rfloor \cdot Q_{C}\right)$, otherwise that will be transported with FCL. By (3) the remaining quantities $\left(Q-\left\lfloor Q / Q_{C}\right\rfloor \cdot Q_{C}\right)$ are also equal to

$$
(d / \theta) \cdot\left\{\left[\exp \left[\theta \cdot\left(T-\left\lfloor T / T_{Q c}\right\rfloor \cdot T_{Q c}\right)\right]-1\right\} .\right.
$$

Therefore the remainder transportation cost is considered as follows:

$$
\min \left\{s \cdot(d / \theta) \cdot\left\{\left[\exp \left[\theta \cdot\left(T-\left\lfloor T / T_{Q c}\right\rfloor \cdot T_{Q c}\right)\right]-1\right\}, C_{F}\right\} .\right.
$$

Then the total transportation cost per cycle $C(T)$ is:

$$
\begin{aligned}
C(T) & =\left\lfloor T / T_{Q c}\right\rfloor C_{F}+\min \left\{\left\{s \cdot(d / \theta) \cdot\left\{\exp \left[\theta \cdot\left(T-\left\lfloor T / T_{Q c}\right\rfloor \cdot T_{Q c}\right)\right]-1\right\}\right\}, C_{F}\right\} \\
& =\min \left\{\left\{\left\lfloor T / T_{Q c}\right\rfloor C_{F}+\left\{s \cdot(d / \theta) \cdot\left\{\exp \left[\theta \cdot\left(T-\left\lfloor T / T_{Q c}\right\rfloor \cdot T_{Q c}\right)\right]-1\right\}\right\},\left[\left(\left\lfloor T / T_{Q c}\right\rfloor+1\right) C_{F}\right]\right\},\right.
\end{aligned}
$$

Let

$$
\left\lfloor T / T_{Q c}\right\rfloor C_{F}+s \cdot(d / \theta) \cdot\left\{\exp \left[\theta \cdot\left(T-\left\lfloor T / T_{Q c}\right\rfloor \cdot T_{Q c}\right)\right]-1\right\}=\left(\left\lfloor T / T_{Q c}\right\rfloor+1\right) C_{F},
$$

then $T=\left\lfloor T / T_{Q c}\right\rfloor \cdot T_{Q c}+\ln \left[(\theta / d) \cdot\left(C_{F} / s\right)+1\right] / \theta$. Therefore we define

$$
\begin{aligned}
\Omega_{1}= & \left\{T \| T / T_{Q c}\right\rfloor \cdot T_{Q c} \leq T \leq\left\lfloor T / T_{Q c}\right\rfloor \cdot T_{Q c} \\
& \left.+\ln \left[(\theta / d) \cdot\left(C_{F} / s\right)+1\right] / \theta\right\}
\end{aligned}
$$

and

$$
\begin{aligned}
\Omega_{2} & =\left\{T \| T / T_{Q c}\right\rfloor \cdot T_{Q c}+\ln \left[(\theta / d) \cdot\left(C_{F} / s\right)+1\right] / \theta \\
& \left.<T<\left(\left\lfloor T / T_{Q c}\right\rfloor+1\right) \cdot T_{Q c}\right\}
\end{aligned}
$$

In $\Omega_{1}$, the transportation cost of remainders with LCL is cheaper than that with a full cargo. In $\Omega_{2}$, remainders are transported with FCL.

Hence $C(T)$ can be reformulated as follows:

$$
C(T)=\left\{\begin{array}{l}
\left\lfloor T / T_{Q c}\right\rfloor C_{F}+\left\{s \cdot(d / \theta) \cdot\left\{\exp \left[\theta \cdot\left(T-\left\lfloor T / T_{Q c}\right\rfloor \cdot T_{Q c}\right)\right]-1\right\}\right\} \text { for } T \in \Omega_{1}, \\
\left(\left\lfloor T / T_{Q c}\right\rfloor+1\right) C_{F} \text { for } T \in \Omega_{2} .
\end{array}\right.
$$


Substituting (6)-(17) into (5), the inventory problem over infinite planning horizon can be converted to prob- lem $A$ as follows:

(Problem A)

$$
\begin{aligned}
\min . P T C(T)= & \left\{\left\{\left(d \times h / \theta^{2}\right)[\exp (\theta T)-\theta T-1]+\{(d / \theta)[\exp (\theta T)-1]-d T\} \times C_{d}\right.\right. \\
& +K+\min \left\{s \cdot ( d / \theta ) \cdot \left\{\left[\exp \left[\theta \cdot\left(T-\left\lfloor T / T_{Q c}\right\rfloor \cdot T_{Q c}\right)\right]-1\right\}+\left\lfloor T / T_{Q}\right\rfloor C_{F},\right.\right. \\
& \left.\left.\left(\left\lfloor T / T_{Q c}\right\rfloor+1\right) C_{F}\right\}\right\} / T
\end{aligned}
$$

subject to $T \geq 0$.

We reformulate $\operatorname{PTC}(T)$ on space $\Omega_{1}$ and $\Omega_{2}$ respectively as follows:

$$
\begin{aligned}
\operatorname{PTC}(T)_{\Omega_{1}}= & \left\{\left(d \times h / \theta^{2}\right)[\exp (\theta T)-\theta T-1]+\{(d / \theta)[\exp (\theta T)-1]-d T\} \times C_{d}+K\right. \\
& +s \cdot(d / \theta) \cdot\left\{\left[\exp \left[\theta \cdot\left(T-\left\lfloor T / T_{Q c}\right\rfloor \cdot T_{Q c}\right)\right]-1\right\}+\left\lfloor T / T_{Q c}\right\rfloor C_{F}\right\} / T
\end{aligned}
$$

and

$$
P T C(T)_{\Omega_{2}}=\left\{\left(d \times h / \theta^{2}\right)[\exp (\theta T)-\theta T-1]+\{(d / \theta)[\exp (\theta T)-1]-d T\} \times C_{d}+K+\left(\left\lfloor T / T_{Q c}\right\rfloor+1\right) C_{F}\right\} / T
$$

After formulating the inventory problem over infinite planning horizon, in the following section, we will discuss about this problem over finite planning horizon.

\subsubsection{Finite Planning Horizon Model}

Suppose the finite planning horizon denote as $T_{H}$. We assume the total order times is $n$ in $T_{H}$ and the corresponding order intervals are $t_{1}, \cdots, t_{i}, \cdots, t_{n}$, respectively. Given the total cost of the whole planning horizon $T_{H}$ is denoted as $T C\left(t_{1}, \cdots, t_{i}, \cdots, t_{n}\right)$. Thus the inven- tory problem over finite planning horizon is formulated as follows:

(Problem B)

$$
\min . T C\left(t_{1}, \cdots, t_{i}, \cdots, t_{n}, n\right)=\sum_{i=1}^{n} T C\left(t_{i}\right)
$$

subject to $\sum_{i=1}^{n} t_{i}=T_{H}$, and $n>0, t_{i}>0$.

Where $T C\left(t_{i}\right)$ is the total cost occurred in $t_{i}$, and according to (18), $T C\left(t_{i}\right)$ can be expressed as follows.

$$
T C\left(t_{i}\right)=\left(d \times h / \theta^{2}\right)\left[\exp \left(\theta t_{i}\right)-\theta t_{i}-1\right]+\left\{(d / \theta)\left[\exp \left(\theta t_{i}\right)-1\right]-d t_{i}\right\} \times C_{d}+K+C\left(t_{i}\right) .
$$

and

$$
C\left(t_{i}\right)=\min \left\{\left\{\left\lfloor t_{i} / T_{Q c}\right\rfloor C_{F}+\left\{s \cdot(d / \theta) \cdot\left\{\exp \left[\theta \cdot\left(t_{i}-\left\lfloor t_{i} / T_{Q c}\right\rfloor \cdot T_{Q c}\right)\right]-1\right\}\right\},\left[\left(\left\lfloor t_{i} / T_{Q c}\right\rfloor+1\right) C_{F}\right]\right\} .\right.
$$

In problem B, we must find the optimal values of $n$ and $t_{i}$ to minimize $T C\left(t_{1}, \cdots, t_{i}, \cdots, t_{n}\right)$.

\subsection{Optimal Ordering Policy}

\subsubsection{Optimal Ordering Policy over the Infinite Planning Horizon}

We first prove that the optimal ordering policies for infinite and finite planning horizon problems as shown in (18) and (21) will satisfy the zero inventory policy in proposition 1.

Proposition 1. The optimal ordering policy for equation (18) and (21) will satisfy the zero inventory policy.

Proof. See appendix A.

We will show $\operatorname{PTC}(T)_{\Omega_{1}}$ and $P T C(T)_{\Omega_{2}}$ are both convex functions in proposition 2. Then we can determine optimal order interval $T_{1}^{*}$ and $T_{2}^{*}$ for the two spaces respectively.

Proposition 2. For a given $\left\lfloor T / T_{Q c}\right\rfloor, \operatorname{PTC}(T)_{\Omega_{1}}$ and $\operatorname{PTC}(T)_{\Omega_{2}}$ are convex functions, i.e., $\partial^{2} \operatorname{PTC}(T)_{\Omega_{1}} / \partial T^{2} \geq 0$ and $\partial^{2} P T C(T)_{\Omega_{2}} / \partial T^{2} \geq 0$.

Proof. See appendix B.

Proposition 3. For a given $\left\lfloor T / T_{Q c}\right\rfloor$, there exist a $T_{1}$ and a $T_{2} \in(0, \infty)$ such that $\left.\frac{\partial P T C(T)_{\Omega_{1}}}{\partial T}\right|_{T_{1}}=0$ and $\left.\frac{\partial P T C(T)_{\Omega_{2}}}{\partial T}\right|_{T_{2}}=0$ for the two spaces respectively, where 


$$
\begin{aligned}
T_{1}= & \operatorname{Arg}_{(T)}\left\{\partial P T C(T)_{\Omega_{1}} / \partial T=0\right\} \\
= & \operatorname{Arg}_{(T)}\left\{\left\{\left\{-K-\left\lfloor T / T_{Q c}\right\rfloor C_{F}+s \cdot(d / \theta) \cdot\left[\exp \left(-\theta \cdot\left[T / T_{Q c}\right\rfloor \cdot T_{Q c}\right)(\theta T-1)\right]\right.\right.\right. \\
& \left.\left.\left.+\left\{d \cdot\left[h+\theta \cdot\left(C_{d}+s\right)\right] / \theta^{2}\right\} \cdot[(\theta T-1) \cdot \exp (\theta T)+1]\right\} / T^{2}\right\}=0\right\}
\end{aligned}
$$

and

$$
\begin{aligned}
T_{2} & =\operatorname{Arg}_{(T)}\left\{\partial P T C(T)_{\Omega_{2}} / \partial T=0\right\} \\
& =\operatorname{Arg}_{(T)}\left\{\left\{\left\{-K-\left(\left\lfloor T / T_{Q c}\right\rfloor+1\right) C_{F}+\left[d\left(h+\theta C_{d}\right) / \theta^{2}\right] \cdot[(\theta T-1) \cdot \exp (\theta T)+1]\right\} / T^{2}\right\}=0\right\}
\end{aligned}
$$

However, when $T_{1}$ or $T_{2}$ is not in its spaces, in such condition, we will consider the end points to replace $T_{1}$ or $T_{2}$.

Proof. See appendix C.

In proposition 2 and 3, $\left\lfloor T / T_{Q c}\right\rfloor$ is assumed known. In order to find the optimal value $T^{*}$ for minimizing $P T C(T)$ in problem A, we first determine the optimal order interval without considering the transportation cost

item $(C(T))$ in $P T C(T)$, denoted as $T_{3}$ by proposition 4 . Then with proposition 5 we show $T^{*}$ is in the interval $\left[\left\lfloor T_{3} / T_{Q c}\right\rfloor T_{Q},\left(\left\lfloor T_{3} / T_{Q c}\right\rfloor+1\right) T_{Q c}\right]$.

Proposition 4. Let $P T C^{\prime}(T)=(T C(T)-C(T)) / T$, there exist a $T_{3} \in(0, \infty)$ such that $\left.\frac{\partial P T C^{\prime}(T)}{\partial T}\right|_{T_{3}}=0$, where

$$
T_{3}=\operatorname{Arg}_{(T)}\left\{\partial P T C^{\prime}(T) / \partial T=0\right\}=\operatorname{Arg}_{(T)}\left\{\left\{\left\{-K+\left[d\left(h+\theta C_{d}\right) / \theta^{2}\right] \cdot[(\theta T-1) \cdot \exp (\theta T)+1]\right\} / T^{2}\right\}=0\right\}
$$

Proof. See appendix D.

Proposition 5. The optimal order interval $T^{*}$ for problem $A$ is in the interval $\left[\left\lfloor T_{3} / T_{Q c}\right\rfloor T_{Q},\left(\left\lfloor T_{3} / T_{Q c}\right\rfloor+1\right) T_{Q c}\right]$.

Proof. See appendix E.

By Proposition 5, $T^{*} \in\left[\left\lfloor T_{3} / T_{Q c}\right\rfloor T_{Q},\left(\left\lfloor T_{3} / T_{Q c}\right\rfloor+1\right) T_{Q c}\right]$. The interval $\left[\left\lfloor T_{3} / T_{Q c}\right\rfloor T_{Q},\left(\left\lfloor T_{3} / T_{Q c}\right\rfloor+1\right) T_{Q c}\right]$ is divided into $\Omega_{1}$ and $\Omega_{2}$ as shown in (15) and (16). By proposition 2 and 3 , we obtain the optimal values of $T_{1}^{*}$ and $T_{2}^{*}$ respectively. Proposition 6 is provided to find optimal order interval $T^{*}$ for problem A.

Proposition 6. There exists an optimal order interval $T^{*}$ in problem A, and $T^{*}$ is determined by the following procedure:

Step 1: Find $T_{3}$ by proposition 4 , then let $\left\lfloor T / T_{Q c}\right\rfloor=\left\lfloor T_{3} / T_{Q c}\right\rfloor$.

Step 2: Find $T_{1}$ by proposition 3 . If

$$
\begin{aligned}
T_{1} \in & {\left[\left\lfloor T_{3} / T_{Q c}\right\rfloor \cdot T_{Q c},\left\lfloor T_{3} / T_{Q c}\right\rfloor \cdot T_{Q c}\right.} \\
& \left.+\ln \left[(\theta / d) \cdot\left(C_{F} / s\right)+1\right] / \theta\right]
\end{aligned}
$$

then set $T_{1}^{*}=T_{1}$ by proposition 2 and 3 , and go to step 4 . Otherwise, go to step 3.

Step 3: Compare $P T C(T)$ for the two end points. Set

$$
\begin{aligned}
T_{1}^{*}= & \arg _{T} \operatorname{mim}\left\{\operatorname{PTC}\left(\left\lfloor T_{3} / T_{Q c}\right\rfloor T_{Q c}\right)_{\Omega_{1}},\right. \\
& \left.\operatorname{PTC}\left(\left(C_{F} / s d\right)+\left\lfloor T_{3} / T_{Q c}\right\rfloor T_{Q c}\right)_{\Omega_{1}}\right\}
\end{aligned}
$$

and go to step 4.
Step 4: Find $T_{2}$ by proposition 3. If

$$
\begin{aligned}
T_{2} \in & {\left[\left\lfloor T_{3} / T_{Q c}\right\rfloor \cdot T_{Q c}+\ln \left[(\theta / d) \cdot\left(C_{F} / s\right)+1\right] / \theta,\right.} \\
& \left.\left(\left\lfloor T_{3} / T_{Q c}\right\rfloor+1\right) \cdot T_{Q c}\right]
\end{aligned}
$$

then set $T_{2}^{*}=T_{2}$ by proposition 2 and 3 , and go to step 6. Otherwise, go to step 5.

Step 5: Compare $P T C(T)$ for the two end points. Set

$$
\begin{aligned}
T_{2}^{*}= & \arg _{T} \operatorname{mim}\left\{\operatorname{PTC}\left(\left(C_{F} / s d\right)+\left\lfloor T_{3} / T_{Q c}\right\rfloor T_{Q c}\right)_{\Omega_{2}},\right. \\
& \left.P T C\left(\left(\left\lfloor T_{3} / T_{Q c}\right\rfloor+1\right) T_{Q c}\right)_{\Omega_{2}}\right\}
\end{aligned}
$$

and go to step 6.

Step 6: Optimal order interval

$$
T^{*}=\arg _{T} \operatorname{mim}\left\{\operatorname{PTC}(T)_{\Omega_{1}}\left(T_{1}^{*}\right), \operatorname{PTC}(T)_{\Omega_{2}}\left(T_{2}^{*}\right)\right\}
$$

\subsubsection{Optimal Ordering Policy over the Finite Planning Horizon}

We first determine the upper bound of optimal order times $n^{*}$ by Proposition 7 .

Proposition 7. The optimal order times $n^{*}$ over finite planning horizon is at most $\left[2 T_{H} / \min \left\{T_{Q c}, T_{3}\right\}\right]$.

Proof. See appendix F.

By proposition 7, the upper bound of order times $n$ is $\left\lceil 2 T_{H} / \min \left\{T_{Q c}, T_{3}\right\} \mid\right.$. Proposition 8 and 9 will show the order intervals over finite planning horizon in the optimal order policy may not be equal, and the difference between any two order intervals is not more than $T_{Q c}$. We also find the lower and upper bounds for the order inter- 
vals.

Proposition 8. Let $t_{i}$ and $t_{j}$ be any two order intervals in the optimal ordering policy, then $\left|t_{i}-t_{j}\right| \leq T_{Q c}$.

Proof. See appendix G.

Proposition 9. Any order interval $t_{i}$ in the optimal ordering policy over finite planning horizon is satisfied

$$
\left\lfloor T /\left(n T_{Q c}\right)\right\rfloor T_{Q c} \leq t_{i} \leq\left(\left\lfloor T /\left(n T_{Q c}\right)\right\rfloor+1\right) T_{Q c} .
$$

Proof. See appendix H.

Proposition 9 shows the lower bound of any order interval in optimal ordering policy is $\left|T /\left(n T_{Q c}\right)\right| T_{Q c}$. Based on proposition 8 and 9, Proposition 10 will show that order intervals in the optimal ordering policy have at most two types.

Proposition 10. Under the given order times $n$, we know the order intervals in the optimal ordering policy have at most two types, $\left\lfloor T /\left(n T_{Q c}\right)\right\rfloor T_{Q c}+t_{L}$ where

$$
0 \leq t_{L} \leq+\ln \left[(\theta / d) \cdot\left(C_{F} / s\right)+1\right] / \theta
$$

and $\left\lfloor T /\left(n T_{Q c}\right)\right\rfloor T_{Q c}+t_{F}$ where

$$
\ln \left[(\theta / d) \cdot\left(C_{F} / s\right)+1\right] / \theta \leq t_{F} \leq T_{Q c} .
$$

Proof. See appendix I.

Based on proposition 8, 9 and 10, we assume that there are $n_{1} \quad\left(0<n_{1}<n\right)$ order times with order interval length $\left\{\left\lfloor T /\left(n T_{Q c}\right)\right\rfloor T_{Q c}+t_{F}\right\}$ and $n-n_{1}$ order times with order interval length $\left\{\left\lfloor T /\left(n T_{Q c}\right)\right\rfloor T_{Q c}+t_{L}\right\}$ over finite planning horizon $T_{H}$ i.e.,

$$
\begin{aligned}
& n_{1}\left(\left\lfloor T_{H} /\left(n T_{Q c}\right)\right\rfloor T_{Q c}+t_{F}\right) \\
& +\left(n-n_{1}\right)\left(\left\lfloor T_{H} /\left(n T_{Q c}\right)\right\rfloor T_{Q c}+t_{L}\right)=T_{H} .
\end{aligned}
$$

Therefore $T C\left(t_{1}, \cdots, t_{i}, \cdots, t_{n}\right)$ can be denoted as $T C\left(n_{1}, n, t_{L}, t_{F}\right)$, and the problem B can be converted to problem B1 as follows:

(Problem B1)

$$
\begin{aligned}
\min & T C\left(n_{1}, n, t_{L}, t_{F}\right)=n_{1}\left\{\left(d \times h / \theta^{2}\right)\left[\exp \left(\theta\left(t_{F}+\left\lfloor T_{H} /\left(n T_{Q c}\right)\right\rfloor T_{Q c}\right)\right)-\theta\left(t_{F}+\left\lfloor T_{H} /\left(n T_{Q c}\right)\right\rfloor T_{Q c}\right)-1\right]\right. \\
& \left.+\left\{(d / \theta)\left[\exp \left(\theta\left(t_{F}+\left\lfloor T_{H} /\left(n T_{Q c}\right)\right\rfloor T_{Q c}\right)\right)-1\right]-d\left(t_{F}+\left\lfloor T_{H} /\left(n T_{Q c}\right)\right\rfloor T_{Q c}\right)\right\} \times C_{d}+K+\left(\left\lfloor T_{H} /\left(n T_{Q c}\right)\right\rfloor+1\right) C_{F}\right\} \\
& +\left(n-n_{1}\right)\left\{\left(d \times h / \theta^{2}\right)\left[\exp \left(\theta\left(t_{L}+\left\lfloor T_{H} /\left(n T_{Q c}\right)\right\rfloor T_{Q c}\right)\right)-\theta\left(t_{L}+\left\lfloor T_{H} /\left(n T_{Q c}\right)\right\rfloor T_{Q c}\right)-1\right]\right. \\
& +\left\{(d / \theta)\left[\exp \left(\theta\left(t_{L}+\left\lfloor T_{H} /\left(n T_{Q c}\right)\right\rfloor T_{Q c}\right)\right)-1\right]-d\left(t_{L}+\left\lfloor T_{H} /\left(n T_{Q c}\right)\right\rfloor T_{Q c}\right)\right\} \times C_{d}+K \\
& \left.+s \cdot(d / \theta) \cdot\left[\exp \left(\theta \cdot t_{L}\right)-1\right]+\left\lfloor T_{H} /\left(n T_{Q c}\right)\right\rfloor C_{F}\right\}
\end{aligned}
$$

subject to

$$
\begin{aligned}
& 0 \leq t_{L} \leq+\ln \left[(\theta / d) \cdot\left(C_{F} / s\right)+1\right] / \theta, \\
& \left\{\ln \left[(\theta / d) \cdot\left(C_{F} / s\right)+1\right] / \theta\right\} \leq t_{F} \leq T_{Q c}, \\
& n_{1}\left(\left[T_{H} /\left(n T_{Q c}\right)\right] T_{Q c}+t_{F}\right) \\
& +\left(n-n_{1}\right)\left(\left[T_{H} /\left(n T_{Q c}\right)\right\rfloor T_{Q c}+t_{L}\right)=T_{H} .
\end{aligned}
$$

In problem $\mathrm{B} 1$, we let

$$
T_{r}=T_{H}-n\left\lfloor T_{H} /\left(n T_{Q c}\right)\right\rfloor T_{Q c},
$$

then $t_{L}$ in problem B1 can expressed as a function of $t_{F}$ as follows:

$$
t_{L}=\left(T_{r}-n_{1} t_{F}\right) /\left(n-n_{1}\right) .
$$

Therefore $T C\left(n_{1}, n, t_{L}, t_{F}\right)$ can be denoted as $T C\left(n_{1}, n, t_{F}\right)$, and problem B1 can be transformed to problem B2 as follows:

(Problem B2)

$$
\begin{aligned}
\min & T C\left(n_{1}, n, t_{F}\right)=n_{1} \cdot\left\{\left(d \times h / \theta^{2}\right)\left[\exp \left(\theta\left(t_{F}+\left\lfloor T_{H} /\left(n T_{Q c}\right)\right\rfloor T_{Q c}\right)\right)-\theta\left(t_{F}+\left\lfloor T_{H} /\left(n T_{Q c}\right)\right] T_{Q c}\right)-1\right]\right. \\
& \left.+\left\{(d / \theta) \cdot\left[\exp \left(\theta\left(t_{F}+\left\lfloor T_{H} /\left(n T_{Q c}\right)\right] T_{Q c}\right)\right)-1\right]-d\left(t_{F}+\left\lfloor T_{H} /\left(n T_{Q c}\right)\right\rfloor T_{Q c}\right)\right\} \times C_{d}\right\} \\
& +\left(n-n_{1}\right) \cdot\left\{( d \times h / \theta ^ { 2 } ) \left\{\operatorname { e x p } \left[\theta \cdot\left\{\left[\left(T_{r}-n_{1} t_{F}\right) /\left(n-n_{1}\right)\right]+\left\lfloor T_{H} /\left(n T_{Q c}\right)\right] T_{Q c}\right\}\right.\right.\right. \\
& \left.-\theta \cdot\left\{\left[\left(T_{r}-n_{1} t_{F}\right) /\left(n-n_{1}\right)\right]+\left\lfloor T_{H} /\left(n T_{Q c}\right)\right] T_{Q c}\right\}-1\right\}+\left\{( d / \theta ) \left[\operatorname { e x p } \left\{\theta \cdot \left\{\left[\left(T_{r}-n_{1} t_{F}\right) /\left(n-n_{1}\right)\right]\right.\right.\right.\right. \\
& \left.\left.\left.\left.+\left\lfloor T_{H} /\left(n T_{Q c}\right)\right] T_{Q c}\right\}-1\right\}-d\left\{\left[\left(T_{r}-n_{1} t_{F}\right) /\left(n-n_{1}\right)\right]+\left\lfloor T_{H} /\left(n T_{Q c}\right)\right] T_{Q c}\right\}\right\} \times C_{d}\right\} \\
& +n K+\left(n \cdot\left\lfloor T_{H} /\left(n T_{Q c}\right)\right\rfloor+n_{1}\right) C_{F}+\left(n-n_{1}\right) \cdot s \cdot(d / \theta) \cdot\left\{\exp \left[\theta \cdot\left(T_{r}-n_{1} t_{F}\right) /\left(n-n_{1}\right)\right]-1\right\}
\end{aligned}
$$


subject to

$$
\begin{aligned}
& \left\{\left(T_{r} / n_{1}\right)-\left[\left(n / n_{1}\right)-1\right] \cdot\left\{\ln \left[(\theta / d) \cdot\left(C_{F} / s\right)+1\right] / \theta\right\}\right\} \\
& \leq t_{F} \leq\left(T_{r} / n_{1}\right),
\end{aligned}
$$

$$
\left\{\ln \left[(\theta / d) \cdot\left(C_{F} / s\right)+1\right] / \theta\right\} \leq t_{F} \leq T_{Q c} .
$$

In Problem B2, the two constraints about $t_{F}$ are combined as follows.

$$
\max \left\{\left\{\left(T_{r} / n_{1}\right)-\left[\left(n / n_{1}\right)-1\right] \cdot\left\{\ln \left[(\theta / d) \cdot\left(C_{F} / s\right)+1\right] / \theta\right\}\right\},\left\{\ln \left[(\theta / d) \cdot\left(C_{F} / s\right)+1\right] / \theta\right\}\right\} \leq t_{F} \leq \min \left\{\left(T_{r} / n_{1}\right), T_{Q c}\right\}
$$

i.e.,

$$
\max \left\{\left\{\left(T_{r}-\left[\left(n-n_{1}\right] \cdot\left\{\ln \left[(\theta / d) \cdot\left(C_{F} / s\right)+1\right] / \theta\right\}\right\}, n_{1} \cdot\left\{\ln \left[(\theta / d) \cdot\left(C_{F} / s\right)+1\right] / \theta\right\}\right\} \leq n_{1} \cdot t_{F} \leq \min \left\{T_{r}, n_{1} \cdot T_{Q c}\right\} .\right.\right.
$$

In (33), if

$$
\max \left\{\left\{\left(T_{r} / n_{1}\right)-\left[\left(n / n_{1}\right)-1\right] \cdot\left\{\ln \left[(\theta / d) \cdot\left(C_{F} / s\right)+1\right] / \theta\right\}\right\},\left\{\ln \left[(\theta / d) \cdot\left(C_{F} / s\right)+1\right] / \theta\right\}\right\}
$$

is larger than $\min \left\{\left(T_{r} / n_{1}\right), T_{Q c}\right\}$, then problem B2 has no feasible solution.

By proposition 11, we will show $T C\left(n_{1}, n, t_{F}\right)$ in problem B2 is a convex function. Then we can determine $t_{F}^{*}$ to find the optimal cost in problem B2.
Proposition 11. For a given $n_{1}$ and $n, T C\left(n_{1}, n, t_{F}\right)$ is a convex function, i.e., $d^{2} T C\left(n_{1}, n, t_{F}\right) / d t_{F}^{2} \geq 0$, and there exist a $t_{F}^{\prime}$ such that $\left.\frac{\partial T C\left(n_{1}, n, t_{F}\right)}{\partial t_{F}}\right|_{t_{F^{\prime}}}=0$, where

$$
\begin{aligned}
\partial T C & \left(n_{1}, n, t_{F}\right) / \partial t_{F}=(d / \theta) \cdot\left\{h \left\{n _ { 1 } \left\{1+\exp \left[\theta \cdot\left(t_{F}+\left\lfloor T_{H} /\left(n T_{Q c}\right)\right] T_{Q c}\right)\right]\right.\right.\right. \\
& \left.-\exp \left[\theta \cdot\left[\left(T_{r}-n_{1} t_{F}\right) /\left(n-n_{1}\right)+\left\lfloor T_{H} /\left(n T_{Q c}\right)\right] T_{Q c}\right]\right\}-n\right\}+\theta n_{1}\left\{C_{d} \exp \left[\theta \cdot\left(t_{F}+\left\lfloor T_{H} /\left(n T_{Q c}\right)\right] T_{Q c}\right)\right]\right. \\
& \left.-\exp \left[\theta \cdot\left[\left(T_{r}-n_{1} t_{F}\right) /\left(n-n_{1}\right)+\left\lfloor T_{H} /\left(n T_{Q c}\right)\right] T_{Q c}\right]\right\}-s \cdot \exp \left[\theta \cdot\left(T_{r}-n_{1} t_{F}\right) /\left(n-n_{1}\right)\right]\right\}=0 .
\end{aligned}
$$

Proof. See appendix J.

Given $n$ and $n_{1}$, we get $t_{F}^{\prime}$ by proposition 11, but $t_{F}^{\prime}$ may not satisfy (32), i.e., the optimal order interval $t_{F}^{*} \quad$ will be considered the two end points of (32). Therefore an algorithm in proposition 12 is provided to find the optimal ordering policy over finite planning horizon.

Proposition 12. The optimal ordering policy over finite planning horizon is determined by the following procedure:

Step 0: Initialize $t_{F}^{*}=0, n^{*}=1, n_{1}^{*}=0$ and $T C^{*}=\infty$. Calculate $T_{Q c}, T_{3}$ (by proposition 4) and $n_{\max }$ (by proposition 7), let $n=1$ and $n_{1}=0$. Go to step 1 .

Step 1: Set $T_{r}=T_{H}-n\left[T_{H} /\left(n T_{Q c}\right)\right] T_{Q c}$, find the lower bound $\mathrm{L}$ and upper bound $\mathrm{U}$ of $t_{F}$ as follows:

$$
\begin{gathered}
L=\max \left\{\left\{\left(T_{r}-\left(n-n_{1}\right) \cdot\left\{\ln \left[(\theta / d) \cdot\left(C_{F} / s\right)+1\right] / \theta\right\}\right\},\right.\right. \\
\left.\left\{n_{1} * \ln \left[(\theta / d) \cdot\left(C_{F} / s\right)+1\right] / \theta\right\}\right\}
\end{gathered}
$$

and $U=\min \left\{T_{r}, n_{1} * T_{Q c}\right\}$. Go to step 2 .

Step 2: Check whether $L \leq U$ is satisfied. If $L \leq U$ is hold, go to step 3, otherwise go to step 5 .

Step 3: Find $t_{F}^{\prime}$ by proposition 11. If $t_{F}^{\prime}$ is not in the interval $[\mathrm{L}, \mathrm{U}]$ then we consider two conditions 1 ) if $n_{1} t_{F}^{\prime}<L$, let $\left.t_{F}^{\prime}=L / n_{1} ; 1\right) n_{1} t_{F}^{\prime}>U$, let $t_{F}^{\prime}=U / n_{1}$.
Go to step 4.

Step 4: Calculate $T C\left(n_{1}, n, t_{F}^{\prime}\right)$. If $T C^{*}>T C\left(n_{1}, n, t_{F}^{\prime}\right)$, then set $T C^{*}>T C\left(n_{1}, n, t_{F}^{\prime}\right), t_{F}^{*}=t_{F}^{\prime}, n_{1}^{*}=n_{1}, n^{*}=n$. Go to step 5 .

Step 5: If $n_{1}<n$, set $n_{1}=n_{1}+1$ and go to step 2, otherwise go to step 6 .

Step 6: If $n<n_{\max }$, set $n=n+1$ and $n_{1}=0$. Go to step 2, otherwise go to step 7 .

Step 7: Print $n^{*}, n_{1}^{*}, t_{F}^{*}$ and $T C^{*}$.

The optimal ordering policy is as follows:

There are $n^{*}$ order times where $n_{1}^{*}$ order times with order interval length $\left\lfloor T_{H} /\left(n^{*} T_{Q c}\right)\right\rfloor T_{Q c} t_{F}^{*}$ and $n^{*}-n_{1}^{*}$ order times with order interval $\left\lfloor T_{H} /\left(n^{*} T_{Q c}\right)\right\rfloor T_{Q c} t_{L}^{*}$, where $t_{L}^{*}=\left(T_{r}-n_{1}^{*} \cdot t_{F}^{*}\right) /\left(n^{*}-n_{1}^{*}\right)$, the optimal cost is $T C^{*}$.

\section{Computational Study}

With two examples given by Rieksts and Ventura [1], we demonstrate our proposed solution procedures for infinite and finite planning horizons with considering deteriorateing item. We will show that the approach proposed by Rieksts and Ventura cannot be applied to deteriorating items. The results for each example are also shown in Table 1. 
Example 1a. (Infinite planning horizon) Suppose the customer demand rate $(d)=2$ units/month, and and the other relevant parameters are given as follows: container maximum capacity $\left(Q_{C}\right)$ is 20 unit, holding cost $(h)=\$$ $1 /$ unit/month, set up cost per order $(K)=200$ /per order, unit container fee $\left(C_{F}\right)=\$ 60.2 /$ unit, and goods transportation cost with LCL (s) is charge by $\$ 3.75 /$ unit per unit good. (The infinite example in [1]).

Example 1b. Data are given by Example 1a, and additional data are given as follows: the deterioration rate $(\theta)$ is 0.05 and the unit deteriorated cost per unit gas volume per unit month $\left(C_{d}\right)$ is $\$ 0.5 /$ unit/month.

With our proposed solution procedure stated in proposition 6, in step 1 we find that $T_{3}=11.484$ by proposition 4 and $\left\lfloor T_{3} / T_{Q c}\right\rfloor=1$. In step 2 , we find that $T_{1}=$ 11.1749 by proposition 3 , and

$$
\begin{aligned}
T_{1} \in & {\left[\left\lfloor T / T_{Q c}\right\rfloor T_{Q c}=8.1093, \ln \left[(\theta / d) \cdot\left(C_{F} / s\right)+1\right] / \theta\right.} \\
& \left.+\left\lfloor T / T_{Q c}\right\rfloor T_{Q c}=14.8578\right],
\end{aligned}
$$

we set $T_{1}^{*}=T_{1}=11.1749$ and go to step 4. In step 4, we find that $T_{2}=13.9267$ by proposition 3 and

$$
\begin{aligned}
T_{2} \notin\left[\ln \left[(\theta / d) \cdot\left(C_{F} / s\right)+1\right] / \theta+\left\lfloor T / T_{Q c}\right\rfloor T_{Q c}\right. \\
\left.=14.8578,\left(\left\lfloor T / T_{Q c}\right\rfloor+1\right) T_{Q c}=16.2186\right],
\end{aligned}
$$

then we go to step 5 . In step 5, we compare $\operatorname{PTC}(T)$ for the two end points in $\Omega_{2}$ and set

$$
\begin{aligned}
T_{2}^{*}= & \arg _{T} \operatorname{mim}\left\{\operatorname{PTC}\left(\left(C_{F} / s d\right)+\left\lfloor T_{3} / T_{Q c}\right\rfloor T_{Q c}\right)_{\Omega_{2}},\right. \\
& \left.\operatorname{PTC}\left(\left(\left\lfloor T_{3} / T_{Q c}\right\rfloor+1\right) T_{Q c}\right){ }_{\Omega_{2}}\right\} \\
= & \arg _{T} \operatorname{mim}\{41.3837,41.9541\}=14.8578
\end{aligned}
$$

then we go to step 6 . In step 6 , optimal order interval

$$
\begin{aligned}
T^{*} & =\arg _{T} \operatorname{mim}\left\{\operatorname{PTC}\left(T_{1}^{*}\right)_{\Omega_{1}}, \operatorname{PTC}\left(T_{2}^{*}\right)_{\Omega_{2}}\right\} \\
& =\arg _{T} \operatorname{mim}\{39.4301,41.3837\}=11.1749
\end{aligned}
$$

and optimal total cost per month is 39.4301 .

Example 2a. (finite planning horizon) Suppose the customer demand rate $(d)=1$ units/month, period of the whole planning horizon $\left(T_{H}\right)=5$ month and the other relevant parameters are given as follows: container maximum capacity $\left(Q_{C}\right)$ is 4 unit, holding cost $(h)=$ $\$ 2 /$ unit/month, set up cost per order $(K)=25 /$ per order, unit container fee $\left(C_{F}\right)=\$ 1000 /$ unit, and goods transportation cost with LCL $(s)$ is charge by $\$ 500 /$ unit per unit good. (The finite example in [1])

Example 2b. Data are given by Example 1a, and additional data are given as follows: the deterioration rate $(\theta)$ is 0.2 and the unit deteriorated cost per unit gas volume per unit time $\left(C_{d}\right)$ is $\$ 0.5 /$ unit/ month.
With our proposed solution procedure in proposition 12 , in step 0 , initialize $t_{F}^{*}=0, n^{*}=1, n_{1}^{*}=0$, $T C^{*}=\infty$, let $n=1$ and $n_{1}=0$. And we find that $T_{Q c}=3.09185, T_{3}=3.95019$ and $n_{\max }=4$ by proposition 4 and 7 , then we go to step 1 . In step 1 , set $T_{r}=5-2.93893 \cdot n\lfloor 1.617 / n\rfloor=2.06107$, we find $U=\min \left\{T_{r}, n_{1} * T_{Q c}\right\}=0$ and

$$
\begin{aligned}
L= & \max \left\{\left\{\left(T_{r}-\left(n-n_{1}\right) \cdot\left\{\ln \left[(\theta / d) \cdot\left(C_{F} / s\right)+1\right] / \theta\right\}\right\},\right.\right. \\
& \left.\left\{n_{1} * \ln \left[(\theta / d) \cdot\left(C_{F} / s\right)+1\right] / \theta\right\}\right\}=0.22579
\end{aligned}
$$

then go to step 2. In step 2, $L>U$, we go to step 5. In step 5, $n_{1}<n$, let $n_{1}=1$ and go to step 1 . In step 1 , $T_{r}=2.06107$ and $L=U=2.06017$. Go to step 2. In step 2, $L=U$, go to step 3. In step 3, we find $n_{1} \cdot t_{F}^{\prime} \notin[L, U]$ by proposition 11, let $t_{F}^{\prime}=L / n_{1}=U / n_{1}=2.06107$. Go to step 4. In step 4 , calculate $\operatorname{TC}\left(\hat{t}_{F}\right)=2062.71$ and $T C^{*}>\operatorname{TC}\left(n_{1}, n, t_{F}^{\prime}\right), T C^{*}=T C\left(\hat{t}_{F_{*}}\right)=2062.71$, $t_{F}^{*}=t_{F}^{\prime}=2.06107, n_{1}^{*}=1$ and $n^{*}=1$. Go to step 5. In step 5, $n_{1}=n$, go to step 6. In step 6, $n<n_{\max }$ and set $n=2$ and go to step 1 . We repeat the algorithm until $n=n_{\max }=4$. At the termination of algorithm, we find $T C^{*}=2062.71, n^{*}=1, n_{1}^{*}=1$ and $t_{F}^{*}=2.06107$, i.e., The optimal ordering policy is: there are 1 order times with order interval length 5 over finite planning horizon $T_{H}=5$ and optimal total cost is 2062.71 .

From Table 1, we know when the deterioration rate $(\theta)$ approach to zero, our results are identical to those obtained by Rieksts and Ventura's model. However in the scenario with considering deteriorating item, Rieksts and Ventura's model is not work well.

In Figure 1 we show the differences of costs per unit time between the two infinite models which increase with the increased value of the deterioration rate $(\theta)$. Similarly, Figure 2 shows the cost differences between the two finite models which increase with the increased value of the deterioration rate $(\theta)$. When the deterioration rate $(\theta)$ is large, Rieksts and Ventura's model does not work well. However, the proposed model can obtain the optimal cost.

\section{Conclusion}

In this paper we propose approaches to resolving the problems of the deteriorating items inventory model with mixed cargo transportation (FCL and LCL) to determine the optimal order interval and to minimize the cost per unit and the total cost over infinite and finite planning horizon. The optimal order intervals over infinite and finite planning horizons are derived and the solution procedures are proposed. Numerical examples are also illustrated. From example, when the deterioration rate $(\theta)$ approaches to zero, our results are identical to those obtained from Rieksts and Ventura's model. The differences 
Table 1. Comparison between this study and Rieksts and Ventura (2008).

\begin{tabular}{|c|c|c|c|}
\hline & & Rieksts \& Ventura (2008) & Proposed model \\
\hline $\begin{array}{c}\text { example 1a } \\
\theta=0, \quad C_{d}=0\end{array}$ & $\begin{array}{l}\text { Optimal solution over infinite } \\
\text { planning horizon }\end{array}$ & $\begin{array}{l}\text { Optimal order interval }=13.6 \\
\text { optimal cost per unit time }=34.7\end{array}$ & $\begin{array}{l}\text { Optimal order interval }=13.6 \\
\text { optimal cost per unit time }=34.7\end{array}$ \\
\hline $\begin{array}{c}\text { example } 1 \mathrm{~b} \\
\theta=0.05, C_{d}=0.5\end{array}$ & $\begin{array}{l}\text { Optimal solution over infinite } \\
\text { planning horizon }\end{array}$ & $\begin{array}{l}\text { With the order interval }=13.6 \text { obtained in } \\
\text { example } 1 \mathrm{a} \text {, the cost per unit time }=40.3358\end{array}$ & $\begin{array}{l}\text { Optimal order interval }=11.1749 \\
\text { Optimal cost per unit time }=39.4301\end{array}$ \\
\hline $\begin{array}{c}\text { example } 2 \mathrm{a} \\
\theta=0, C_{d}=0\end{array}$ & $\begin{array}{l}\text { Optimal solution over finite } \\
\text { planning horizon }\end{array}$ & 1 order interval $=5$. optimal cost $=1050$ & $\begin{array}{l}1 \text { order interval }=5 \\
\text { the optimal cost }=1050\end{array}$ \\
\hline $\begin{array}{c}\text { example 2b } \\
\theta=0.2, \quad C_{d}=0.5\end{array}$ & $\begin{array}{l}\text { Optimal solution over finite } \\
\text { planning horizon }\end{array}$ & $\begin{array}{l}\text { With } 1 \text { order interval }=5 \text { obtained in example } \\
2 a \text {, the cost }=2338.1\end{array}$ & $\begin{array}{l}1 \text { order interval }=5 \\
\text { the optimal cost }=2062.71\end{array}$ \\
\hline
\end{tabular}

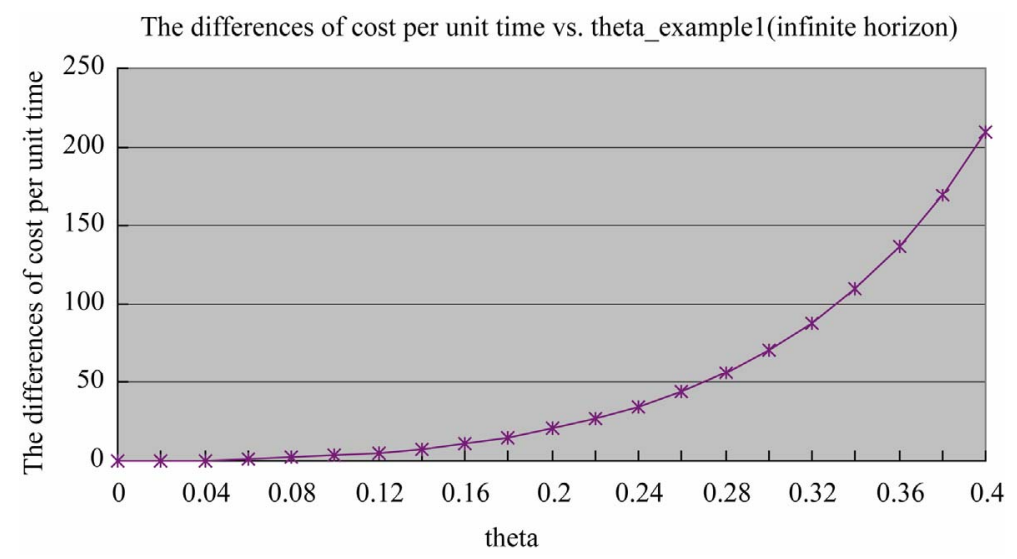

Figure 1. The differences of cost per unit time vs. the deterioration rate (in example 1a and 1b: infinite planning horizon case).

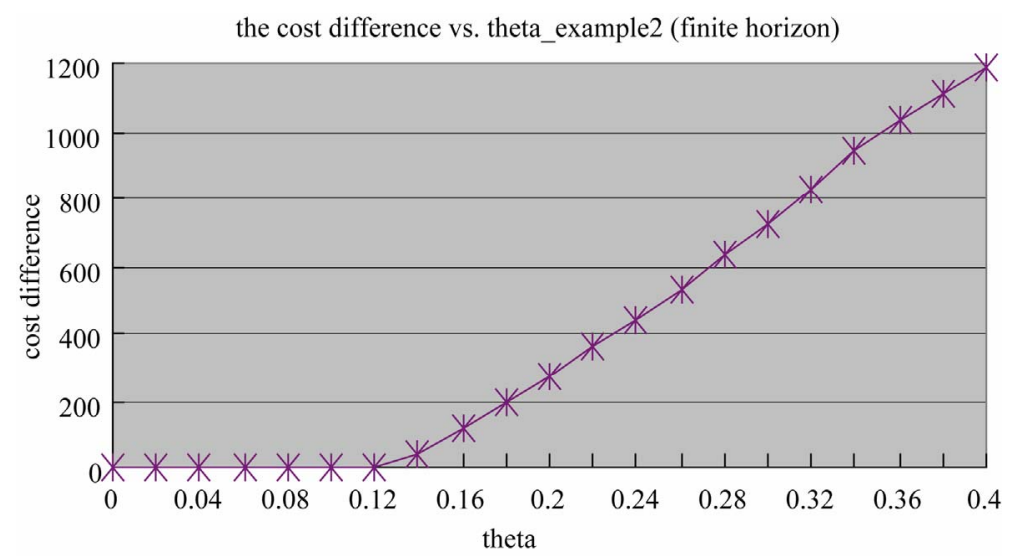

Figure 2. The differences of cost vs. the deterioration rate (in example 2a and $2 \mathrm{~b}$ : finite planning horizon case).

of costs between the two models increase with the rising deterioration rate $(\theta)$. The results of the proposed model perform better compared to the inventory model proposed by Rieksts and Ventura [1] when deterioration rate $(\theta)$ is large. It would be of interest to extend the model to situations where the retailer receives random demand before the customer's real demand occurs.

\section{REFERENCES}

[1] B. Rieksts and J. Ventura, “Optimal Inventory Policies with Two Modes of Freight Transportation,” European Journal of Operational Research, Vol. 186, No. 2, 2008, pp. 576-585. doi:10.1016/j.ejor.2007.01.042

[2] S. K. Goyal and B. C. Giri, "Recent Trends in Modeling of Deteriorating Inventory," European Journal of Operational Research, Vol. 134, No. 1, 2001, pp. 1-16. doi:10.1016/S0377-2217(00)00248-4

[3] R. Li, H. Lan and J. R. Mawhinney, "A Review on Deteriorating Inventory Study," Journal of Service Science and Management, Vol. 3, No. 1, 2010, pp. 117-129. doi:10.4236/jssm.2010.31015

[4] B. Rieksts and J. Ventura, “Two-Stage Inventory Models 
with a Bi-Modal Transportation Cost," Computers and Operations Research, Vol. 37, No. 1, 2010, pp. 20-31. doi:10.1016/j.cor.2009.02.026

[5] D. Aucamp, "Nonlinear Freight Costs in the EOQ Problem,” European Journal of Operational Research, Vol. 9, No. 1, 1982, pp. 61-63. doi:10.1016/0377-2217(82)90011-X

[6] S. Lippman, "Economic Order Quantities and Multiple Set-Up Costs," Management Science, Vol. 18, No. 1, 1971, pp. 39-47. doi:10.1287/mnsc.18.1.39

[7] C. Y. Lee, "The Economic Order Quantity for Freight Discount Costs,” IIE Transactions, Vol. 18, No. 3, 1986, pp. 318-320. doi:10.1080/07408178608974710

[8] H. Hwang, D. Moon and S. Shinn, “An EOQ Model with Quantity Discounts for both Purchasing Price and Freight Cost," Computers and Operations Research, Vol. 17, No. 1, 1990, pp. 73-78. doi:10.1016/0305-0548(90)90029-7

[9] R. Tersine, S. Barman and R. Toelle, "Composite lot Sizing with Quantity and Freight Discounts," Computers and Industrial Engineering, Vol. 28, No. 1, 1995, pp. 107-122. doi:10.1016/0360-8352(94)00031-H

[10] W. Adelwahab and M. Sargious, "Freight Rate Structure and Optimal Shipment Size in Freight Transportation," Logistics and Transportation Review, Vol. 26, No. 3, 1990, pp. 271-292.

[11] S. Swenseth and M. Godfrey, "Incorporating Transportation Costs into Inventory Replenishment Decisions," International Journal of Production Economics, Vol. 77, No. 2, 2002, pp. 113-130. doi:10.1016/S0925-5273(01)00230-4

[12] L. Burns, R. Hall and D. Blumenfeld, "Distribution Strategies That Minimize Transportation and Inventory Costs," Operations Research, Vol. 33, No. 3, 1985, pp. 469-490. doi:10.1287/opre.33.3.469

[13] P. Larson, "The Economic Transportation Quantity," Transportation Journal, Vol. 28, No. 2, 1988, pp. 43-48.

[14] K.-J. Chung and C.-N. Lin, “Optimal Inventory Replenishment Models for Deteriorating Items Taking Account of Time Discounting," Computers and Operations Research, Vol. 28, No. 1, 2001, pp. 67-83. doi:10.1016/S0305-0548(99)00087-8

[15] L. Benkherouf, A. Boumenir and L. Aggoun, "A Diffusion Inventory Model for Deteriorating Items,” Applied Mathematics and Computation, Vol. 138, No. 1, 2003, pp. 21-39. doi:10.1016/S0096-3003(02)00097-8

[16] K. J. Heng, J. Labban and R. L. Lim, “An Order-Level Lot Size Inventory Model for Deteriorating Items with Finite Replenishment Rate," Computer and Industrial Engineering, Vol. 20, No. 2, 1991, pp. 187-197. doi:10.1016/0360-8352(91)90024-Z

[17] F. Raafat, P. M. Wolfe and H. K. Eldin, “An Inventory Model for Deteriorating Items," Computers and Industrial Engineering, Vol. 20, No. 2, 1991, pp. 89-94. doi:10.1016/0360-8352(91)90043-6

[18] C. H. Goh, B. S. Greenberg and H. Matsuo, "Two-Stage Perishable Inventory Models,” Management Science, Vol. 39, No. 5, 1993, pp. 633-649. doi:10.1287/mnsc.39.5.633
[19] H. Yan and T. C. E. Cheng, “Optimal Production Stopping and Restarting Times for an EOQ Model with Deterioration Items," Journal of the Operational Research Society, Vol. 49, No. 12, 1998, pp. 1288-1295.

[20] B. C. Giri, T. Chakrabarty and K. S. Chaudhuri, "A Note on a Lot Sizing Heuristic for Deteriorating Items with Time Varying Demands and Shortages,” Computers and Operations Research, Vol. 27, No. 6, 2000, pp. 495-505. doi:10.1016/S0305-0548(99)00013-1

[21] J.-T. Teng, H.-J. Chang, C.-Y. Dye and C.-H. Hung, “An Optimal Replenishment Policy for Deteriorating Items with Time-Varying Demand and Partial Backlogging," Operations Research Letters, Vol. 30, No. 6, 2002, pp. 387-393. doi:10.1016/S0167-6377(02)00150-5

[22] K.-J. Chung, P. Chu and S.-P. Lan, “A Note on EOQ Models for Deteriorating Items under Stock Dependent Selling Rate,” European Journal of Operational Research, Vol. 124, No. 3, 2000, pp. 550-559. doi:10.1016/S0377-2217(99)00203-9

[23] B. C. Giri and K. S. Chaudhuri, "Deterministic Models of Perishable Inventory with Stock-Dependent Demand Rate and Nonlinear Holding Cost," European Journal of Operational Research, Vol. 105, No. 3, 1998, pp. 467-474. doi:10.1016/S0377-2217(97)00086-6

[24] D. K. Bhattachaya, "Production, Manufacturing and Logistics on Multi-Item Inventory," European Journal of Operational Research, Vol. 162, No. 3, 2005, pp. 786791.

[25] K.-S. Wu, L.-Y. Ouyang and C.-T. Yang, “An Optimal Replenishment Policy for Non-Instantaneous Deteriorating Items with Stock-Dependent Demand and Partial Backlogging," International Journal of Production Economics, Vol. 101, No. 2, 2006, pp. 369-384. doi:10.1016/j.ijpe.2005.01.010

[26] H. M. Wee, “A Replenishment Policy for Items with a Price-Dependent Demand and a Varying Rate of Deterioration," Production Planning and Control, Vol. 8, No. 5, 1997, pp. 494-499. doi:10.1080/095372897235073

[27] H.-M. Wee and S.-T. Law, "Economic Production Lot Size for Deteriorating Items Taking Account of the TimeValue of Money," Computers and Operations Research, Vol. 26, No. 6, 1999, pp. 545-558. doi:10.1016/S0305-0548(98)00078-1

[28] P. M. Ghare and G. P. Schrader, "A Model for an Exponentially Decaying Inventory,” Journal of Industrial Engineering, Vol. 14, No. 5, 1963, pp. 238-243.

[29] Y. K. Shah and M. C. Jaiswal, “An Order-Level Inventory Model for a System with Constant Rate of Deterioration,” Opsearch, Vol. 14, 1977, pp. 174-184.

[30] G. Padmanabhana and P. Vratb, "EOQ Models for Perishable Items under Stock Dependent Selling Rate," European Journal of Operational Research, Vol. 86, No. 2, 1995, pp. 281-292. doi:10.1016/0377-2217(94)00103-J

[31] A. K. Bhunia and M. Maiti, “An Inventory Model of Deteriorating Items with Lot-Size Dependent Replenishment Cost and a Linear Trend in Demand," Applied Mathematical Modeling, Vol. 23, No. 4, 1999, pp. 301-308. doi:10.1016/S0307-904X(98)10089-6 
[32] A. K. Bhunia and M. Maiti, "Deterministic Inventory Model for Deteriorating Items with Finite Rate of Replenishment Dependent on Inventory Level,” Computers and Operations Research, Vol. 25, No. 11, 1998, pp. 9971006. doi:10.1016/S0305-0548(97)00091-9

[33] P. L. Abad, “Optimal Price and Order Size for a Reseller under Partial Backordering," Computers and Operations Research, Vol. 28, No. 1, 2001, pp. 53-65. doi:10.1016/S0305-0548(99)00086-6

[34] S. Mukhopadhyay, R. N. Mukherjee and K. S. Chaudhuri, "Joint Pricing and Ordering Policy for a Deteriorating Inventory," Computers and Industrial Engineering, Vol.
47, No. 4, 2004, pp. 339-349. doi:10.1016/j.cie.2004.06.007

[35] N. K. Mahapatra, "Decision Process for Multi-Objective, Multi-Item Production-Inventory System via Interactive Fuzzy Satisficing Technique," Computers and Mathematics with Applications, Vol. 49, No. 5, 2005, pp. 805821. doi:10.1016/j.camwa.2004.07.020

[36] L. Schwarz, "Economic Order Quantities for Products with Finite Demand Horizons,” AIIE Transactions, Vol. 4, No. 3, 1972, pp. 234-237.

doi:10.1080/05695557208974855 


\section{Appendix A}

Proof of Proposition 1: Rieksts and Ventura [1] presented proofs similar to proposition 1 and proposition $7-10$. Proof is given by contradiction. Let $I(t)$ denote the inventory level at time $t$ and $Q(t)$ denote the quantity ordered at time $t$. Suppose there is an optimal ordering policy that does not satisfy the zero-inventory ordering property at time $t^{\prime}$ that is the order time closest to time $T_{H}$ and $I\left(t^{\prime}\right)>0$. We consider another ordering policy at time $\left(t^{\prime}+I\left(t^{\prime}\right) / d\right)$. The holding cost of this adjusted policy is reduced by $h Q\left(t^{\prime}\right) I\left(t^{\prime}\right) / d$. This contradicting the optimality of ordering policy, therefore the optimal ordering policy over finite planning horizon will satisfy the zero inventory policy. The similar argument can extend to infinite planning horizon.

\section{Appendix B}

Proof of Proposition 2:

$$
\begin{aligned}
\partial^{2} P T C(T)_{\Omega} / \partial T^{2}= & \left\{2\left(K+\left\lfloor T / T_{Q c}\right\rfloor C_{F}\right) \cdot \theta^{2}+s \cdot\left\{\exp \left[\theta \cdot\left(T-\left\lfloor T / T_{Q c}\right\rfloor T_{Q c}\right)\right] \cdot\left[(\theta T-1)^{2}+1\right]-2\right\}\right. \\
& +d h\left\{\exp (\theta T) \cdot\left[(\theta T-1)^{2}+1\right]-2\right\}+\left\{C_{d} \theta\left\{\exp (\theta T) \cdot\left[(\theta T-1)^{2}+1\right]-2\right\}\right\} / T^{3} \theta^{2}
\end{aligned}
$$

Let

$$
\begin{aligned}
F(T)= & 2\left(K+\left\lfloor T / T_{Q c}\right\rfloor C_{F}\right) \cdot \theta^{2}+s \cdot\left\{\exp \left[\theta \cdot\left(T-\left\lfloor T / T_{Q c}\right\rfloor T_{Q c}\right)\right] \cdot\left[(\theta T-1)^{2}+1\right]-2\right\} \\
& +d h\left\{\exp (\theta T) \cdot\left[(\theta T-1)^{2}+1\right]-2\right\}+\left\{C_{d} \theta\left\{\exp (\theta T) \cdot\left[(\theta T-1)^{2}+1\right]-2\right\}\right. \\
F^{\prime}(T)= & d \cdot T^{2} \cdot \theta^{3} \cdot\left\{s \cdot \theta \cdot \exp \left[\theta \cdot\left(T-\left\lfloor T / T_{Q c}\right\rfloor T_{Q c}\right)\right]+\exp (\theta T) \cdot\left(h+C_{d} \cdot \theta\right)\right\}>0,
\end{aligned}
$$

therefore $F(T)$ is a increasing function and $\left.F(0)=\left(K+C_{F} \cdot \mid T / T_{Q c}\right\rfloor\right) \theta>0$, i.e., $F(T) \geq 0$

Therefore $\partial^{2} P T C(T)_{\Omega_{1}} / \partial T \geq 0$. A similar argument also shows that $\partial^{2} P T C(T)_{\Omega_{2}} / \partial T \geq 0$.

\section{Appendix C}

Proof of Proposition 3: Redefine $\partial^{2} P T C(T)_{\Omega_{1}} / \partial T=R(T), \quad R\left(0^{+}\right)=-\infty<0$ and $R(\infty)=\infty>0$. By the intermediate value theorem, there exist a $T_{1} \in(0, \infty)$ such that $\left.\frac{\partial P T C(T)_{\Omega_{1}}}{\partial T}\right|_{T_{1}}=0$. i.e.,

$$
\begin{aligned}
T_{1}= & \operatorname{Arg}_{(T)}\left\{\partial P T C(T)_{\Omega_{1}} / \partial T=0\right\} \\
= & \operatorname{Arg}_{(T)}\left\{\left\{\left\{-K-\left\lfloor T / T_{Q c}\right\rfloor C_{F}+s \cdot(d / \theta)\right.\right.\right. \\
& \cdot\left[\exp \left(-\theta \cdot\left\lfloor T / T_{Q c}\right\rfloor \cdot T_{Q c}\right)(\theta T-1)\right] \\
& +\left\{d \cdot\left[h+\theta \cdot\left(C_{d}+s\right)\right] / \theta^{2}\right\} \\
& \left.\left.\cdot[(\theta T-1) \cdot \exp (\theta T)+1]\} / T^{2}\right\}=0\right\}
\end{aligned}
$$

Redefine $\partial P T C(T)_{\Omega_{2}} / \partial T=R(T), R\left(0^{+}\right)=-\infty<0$ and $R(\infty)=\infty>0$. By the intermediate value theorem, there exist a $T_{2} \in(0, \infty)$ such that $\left.\frac{\partial P T C(T)_{\Omega_{2}}}{\partial T}\right|_{T_{2}}=0$. i.e.,

$$
\begin{aligned}
& T_{2}=\operatorname{Arg}_{(T)}\left\{\partial P T C(T)_{\Omega_{2}} / \partial T=0\right\} \\
& =\operatorname{Arg}_{(T)}\left\{\left\{\left\{-K-\left(\left\lfloor T / T_{Q c}\right\rfloor+1\right) C_{F}\right.\right.\right. \\
& \left.\left.\left.+\left[d\left(h+\theta C_{d}\right) / \theta^{2}\right] \cdot[(\theta T-1) \cdot \exp (\theta T)+1]\right\} / T^{2}\right\}=0\right\}
\end{aligned}
$$

Therefore we can find $T_{1}$ in $\Omega_{1}$ and $T_{2}$ in $\Omega_{2}$ by setting $\partial P T C(T)_{\Omega_{1}} / \partial T=0$ and $\partial P T C(T)_{\Omega_{2}} / \partial T=0$.

\section{Appendix D}

Proof of Proposition 4: We get

$$
\begin{array}{r}
\partial P T C^{\prime}(T) / \partial T=\left\{\left(d \times h / \theta^{2}\right)[\exp (\theta T)-\theta T-1]\right. \\
\left.+\{(d / \theta)[\exp (\theta T)-1]-d T\} \times C_{d}+K\right\} / T
\end{array}
$$

and

$$
\begin{aligned}
\partial P T C^{\prime}(T) / \partial T^{2}=\left(K / T^{3}\right)+\left\{\left[d\left(h+\theta C_{d}\right) / \theta^{2}\right]\right. \\
\left.\cdot\left\{\left[(\theta T-1)^{2}+1\right] \cdot \exp (\theta T)-2\right\} / T^{3}\right\} \geq 0,
\end{aligned}
$$

therefore $P T C^{\prime}(T)$ is a convex function. Redefine $\partial P T C^{\prime}(T) / \partial T=R(T)$, we find $R\left(0^{+}\right)=-\infty<0$ and $R(\infty)=\infty>0$. By the intermediate value theorem, there exist a $T_{3} \in(0, \infty)$ such that $\left.\frac{\partial P T C(T)_{\Omega_{2}}}{\partial T}\right|_{T_{2}}=0$. Where 


$$
\begin{aligned}
T_{3}= & \operatorname{Arg}_{(T)}\left\{\partial P T C^{\prime}(T) / \partial T=0\right\} \\
= & \operatorname{Arg}_{(T)}\left\{\left\{\left\{-K+\left[d\left(h+\theta C_{d}\right) / \theta^{2}\right]\right.\right.\right. \\
& \left.\left.\cdot[(\theta T-1) \cdot \exp (\theta T)+1]\} / T^{2}\right\}=0\right\}
\end{aligned}
$$

Therefore we can find $T_{3} \in(0, \infty)$ that is the optimal order interval that minimize $P T C^{\prime}(T)$.

\section{Appendix E}

Proof of Proposition 5: The statement $\left\lfloor T / T_{Q c}\right\rfloor=\left\lfloor T_{3} / T_{Q c}\right\rfloor$ is equivalent to the statement

$$
\left\lfloor T / T_{Q c}\right\rfloor T_{Q c} \leq T_{3} \leq\left(\left\lfloor T_{3} / T_{Q c}\right\rfloor+1\right) T_{Q c} .
$$

Proof by contradiction. $T_{3}$ is the optimal order interval that minimize $P T C^{\prime}(T)$ (defined in proposition 4). Only the average holding and setup costs included in $P T C^{\prime}(T)$ represent a convex function with a minimum in $T_{3}$.

Suppose that the optimal order interval $T^{*}$ of $P T C(T)$ is not on the interval

$$
\left[\left\lfloor T / T_{Q c}\right\rfloor T_{Q c},\left(\left\lfloor T / T_{Q c}\right\rfloor+1\right) T_{Q c}\right]
$$

where

$$
\left\lfloor T / T_{Q c}\right\rfloor T_{Q c} \leq T_{3} \leq\left(\left\lfloor T / T_{Q c}\right\rfloor+1\right) T_{Q c} .
$$

We first consider $T^{*}>\left(\left\lfloor T / T_{Q c}\right\rfloor+1\right) T_{Q c}$. Since the average holding and setup costs represent a convex function with a minimum on the interval

$\left[\left\lfloor T / T_{Q c}\right\rfloor T_{Q c},\left(\left\lfloor T / T_{Q c}\right\rfloor+1\right) T_{Q c}\right]$, the average holding cost and setup costs for $T^{*}$ are more than for

$\left(\left\lfloor T / T_{Q c}\right\rfloor+1\right) T_{Q c}$. The average transportation cost at

$\left(\left\lfloor T / T_{Q c}\right\rfloor+1\right) T_{Q c}$ is at a minimum since full cargo loads are used. This implies that the average cost at $T^{*}$ is greater than the cost at $\left(\left\lfloor T / T_{Q c}\right\rfloor+1\right) T_{Q c}$. A similar argument also shows that the cost at $T^{*}$ is greater than the cost at $\left\lfloor T / T_{Q c}\right\rfloor T_{Q c}$. This contradicting the optimality of $T^{*}$, therefore the optimal order interval $T^{*}$ of $\operatorname{PTC}(T)$ is in the range $\left[\left\lfloor T / T_{Q c}\right\rfloor T_{Q c},\left(\left\lfloor T / T_{Q c}\right\rfloor+1\right) T_{Q c}\right]$ where $\left\lfloor T / T_{Q c}\right\rfloor T_{Q c} \leq T_{3} \leq\left(\left\lfloor T / T_{Q c}\right\rfloor+1\right) T_{Q c}$.

\section{Appendix $\mathbf{F}$}

Proof of Proposition 7: Proof is given by contradiction. Suppose that there are $\left\{\left[2 T_{H} / \min \left\{T_{Q c}, T_{3}\right\}\right]+1\right\}$ order times in the optimal ordering policy. This implies that there are at least two order intervals, $t^{\prime \prime}$ and $t^{\prime \prime \prime}$ such that $0<t^{\prime \prime} \leq t^{\prime \prime \prime} \leq \min \left\{T_{Q c}, T_{3}\right\} / 2$. Consider a ordering policy that is identical to the optimal ordering policy except that the order intervals $t^{\prime \prime}$ and $t^{\prime \prime \prime}$ are combined into a single order interval. Because the average holding and setup costs included in $P T C^{\prime}(T)$ represent a convex function with a minimum in $T_{3}$ and $t^{\prime \prime} \leq t^{\prime \prime \prime}<t^{\prime \prime}+t^{\prime \prime \prime} \leq T_{3}$, the average holding and setup cost the adjusted policy is lower than the optimal ordering policy. Otherwise the average transportation cost is not increased in the adjusted policy since

$t^{\prime \prime} \leq t^{\prime \prime \prime}<t^{\prime \prime}+t^{\prime \prime \prime} \leq T_{Q c}$. Thus the total cost of the adjusted policy is less than the optimal ordering policy. This contradicting the optimality of ordering policy, therefore the optimal order times over finite planning horizon is at most $\left[2 T_{H} / \min \left\{T_{Q c}, T_{3}\right\}\right]$.

\section{Appendix G}

Proof of Proposition 8: Proof is given by contradiction. Suppose that the optimal ordering policy has at least two order intervals $t^{\prime \prime}$ and $t^{\prime \prime \prime}$ such that $\left|t^{\prime \prime}-t^{\prime \prime \prime}\right|>T_{Q c}$. We consider another ordering policy that is analogous to the optimal ordering policy with two order intervals

$\tilde{t}=t^{\prime \prime}+T_{Q c}$ and $\bar{t}=t^{\prime \prime \prime}-T_{Q c}$. In the adjusted policy, the set up cost and transportation cost are kept unchanged, but the holding cost of the adjusted policy is reduced by $h d T_{Q c}\left(t^{\prime \prime}-t^{\prime}-T_{Q c}\right)$. This contradicting the optimality of ordering policy, therefore $\left|t_{i}-t_{j}\right| \leq T_{Q c}$.

\section{Appendix $\mathbf{H}$}

Proof of Proposition 9: Proof is given by contradiction. Suppose that there exist an order interval $t_{i} \leq\left\lfloor T_{H} /\left(n T_{Q c}\right)\right\rfloor T_{Q c}$ in the optimal ordering policy. Let $t_{j}>\left(T_{H} / n\right)$ for arbitrarily $j$ and $m$ be the cardinality of the set $\left\{j \mid t_{j}>\left(T_{H} / n\right)\right\}$. Because $t_{i} \leq\left\lfloor T_{H} /\left(n T_{Q c}\right)\right\rfloor T_{Q c}$ and $\left|t_{i}-t_{j}\right| \leq T_{Q c} \quad$ (proposition 8), $\left\lfloor T_{H} /\left(n T_{Q c}\right)\right\rfloor T_{Q c}-T_{Q c} \leq t_{i}$. In two cases,

$$
\text { 1) } \begin{aligned}
\ln & {\left[(\theta / d) \cdot\left(C_{F} / s\right)+1\right] / \theta } \\
& <t_{i}-\left(\left\lfloor T_{H} /\left(n T_{Q c}\right)\right\rfloor T_{Q c}-T_{Q c}\right) \leq T_{Q c} \text { and } \\
\text { 2) } & 0 \leq t_{i}-\left(\left\lfloor T_{H} /\left(n T_{Q c}\right)\right\rfloor T_{Q c}-T_{Q c}\right) \\
& <\ln \left[(\theta / d) \cdot\left(C_{F} / s\right)+1\right] / \theta
\end{aligned}
$$

considering another ordering policy that is analogous to the optimal ordering policy with two order intervals $\tilde{t}$ and $\bar{t}$. For case 1), let $\tilde{t}=\left\lfloor T_{H} /\left(n T_{Q c}\right)\right\rfloor T_{Q c}$ and $\bar{t}=t_{j}-\left(\left\lfloor T_{H} /\left(n T_{Q c}\right)\right\rfloor T_{Q c}-t_{i}\right) / m$. Because the average holding and setup costs in cost function is a convex function of $t$ and $t_{i}<\tilde{t} \leq \bar{t}<t_{j}$, then the holding cost and set up cost of the adjusted policy (order intervals $\tilde{t}, \bar{t}$ ) is less than the policy $\left(t_{i}, t_{j}\right)$. And transportation cost are kept unchanged since 


$$
\begin{aligned}
& \ln \left[(\theta / d) \cdot\left(C_{F} / s\right)+1\right] / \theta \\
& <t_{i}-\left(\left\lfloor T_{H} /\left(n T_{Q c}\right)\right] T_{Q c}-T_{Q c}\right) \leq T_{Q c}
\end{aligned}
$$

and $\tilde{t}=\left|T_{H} /\left(n T_{\mathrm{Qc}}\right)\right| T_{\mathrm{Qc}}$. Hence the total cost of the adjusted policy is less than the optimal ordering policy in case 1). For case 2), let $\tilde{t}=\left\lfloor T_{H} /\left(n T_{Q c}\right)\right\rfloor T_{Q c}$ and

$$
\bar{t}=\sum_{j} t_{j} / m-\left(\left\lfloor T_{H} /\left(n T_{Q c}\right)\right\rfloor T_{Q c}-t_{i}\right) / m,
$$

$j \in\left\{j \mid t_{j}>\left(T_{H} / n\right)\right\} \quad m$ be the cardinality of the set

$\left\{j \mid t_{j}>\left(T_{H} / n\right)\right\}$. Because $t_{i}<\tilde{t} \leq \bar{t}<\sum_{j} t_{j} / m$, then the holding cost and set up cost of the adjusted policy is reduced due to equality of order intervals. And transportation cost are kept unchanged since

$$
0 \leq \sum_{j} t_{j} / m-\left\lfloor T_{H} /\left(n T_{Q c}\right)\right\rfloor T_{Q c}<\ln \left[(\theta / d) \cdot\left(C_{F} / s\right)+1\right] / \theta .
$$

Hence the total cost of the adjusted policy is less than the optimal ordering policy in case (2). This contradicts the optimality of ordering policy. Similar argument is also hold in $t_{i} \leq\left(\left|T_{H} /\left(n T_{Q c}\right)\right|+1\right) T_{Q c}$. Therefore, if $t_{i}$ is an optimal order interval with $n$ order times,

$$
\left\lfloor T_{H} /\left(n T_{Q c}\right)\right\rfloor T_{Q c} \leq t_{i} \leq\left(\left\lfloor T_{H} /\left(n T_{Q c}\right)\right\rfloor+1\right) T_{Q c} .
$$

\section{Appendix I}

Proof of Proposition 10: Proposition 10 is equivalent to the statement as follows: $t^{\prime}$ and $t^{\prime \prime}$ are any two order intervals in an optimal ordering policy. If 1): $t^{\prime}$ and $t^{\prime \prime}$ fall in

$$
\begin{aligned}
& \left(\left\lfloor T_{H} /\left(n T_{Q c}\right)\right\rfloor T_{Q c},\right. \\
& \left.\ln \left[(\theta / d) \cdot\left(C_{F} / s\right)+1\right] / \theta+\left\lfloor T_{H} /\left(n T_{Q c}\right)\right\rfloor T_{Q c}\right)
\end{aligned}
$$

simultaneously or 2) $t^{\prime}$ and $t^{\prime \prime}$ fall in

$$
\begin{aligned}
& \left(\ln \left[(\theta / d) \cdot\left(C_{F} / s\right)+1\right] / \theta+\left\lfloor T_{H} /\left(n T_{Q c}\right)\right\rfloor T_{Q c},\right. \\
& \left.\left(\left\lfloor T_{H} /\left(n T_{Q c}\right)\right\rfloor+T_{Q c}\right)\right)
\end{aligned}
$$

simultaneously, then $t^{\prime}=t^{\prime \prime}$.

Proof is given by contradiction. Suppose that two order intervals $t^{\prime}$ and $t^{\prime \prime}$ in the optimal ordering policy is such that

$$
t^{\prime}-j T_{Q c}<t^{\prime \prime}-j T_{Q c}<\ln \left[(\theta / d) \cdot\left(C_{F} / s\right)+1\right] / \theta,
$$

where $j=\left\lfloor T_{H} /\left(n T_{Q c}\right)\right\rfloor$ (by proposition 9). We consider another ordering policy that is analogous to the optimal ordering policy but two order intervals $\tilde{t}=\left(t^{\prime}+t^{\prime \prime}\right) / 2$ and $\bar{t}=\left(t^{\prime}+t^{\prime \prime}\right) / 2$.

$$
t^{\prime}-j T_{Q c}<t^{\prime \prime}-j T_{Q c}<\ln \left[(\theta / d) \cdot\left(C_{F} / s\right)+1\right]
$$

implies that

$$
\left(t^{\prime}+t^{\prime \prime}\right) / 2-j T_{Q c}<\ln \left[(\theta / d) \cdot\left(C_{F} / s\right)+1\right] / \theta .
$$

In the adjusted policy, the set up cost and transportation cost are kept unchanged, but the holding cost of the adjusted policy is reduced due to equality of order intervals (see Schwarz [36]). This contradicting the optimality of ordering policy, and similar proof is also proved in

$$
t^{\prime}-j T_{Q c}>t^{\prime \prime}-j T_{Q c}>\ln \left[(\theta / d) \cdot\left(C_{F} / s\right)+1\right] / \theta .
$$

Therefore if $t^{\prime}$ and $t^{\prime \prime}$ fall in

$$
\begin{aligned}
& \left(\left\lfloor T_{H} /\left(n T_{Q c}\right)\right\rfloor T_{Q c},\right. \\
& \left.\ln \left[(\theta / d) \cdot\left(C_{F} / s\right)+1\right] / \theta+\left\lfloor T_{H} /\left(n T_{Q c}\right)\right\rfloor T_{Q c}\right)
\end{aligned}
$$

simultaneously or (2) $t^{\prime}$ and $t^{\prime \prime}$ fall in

$$
\begin{aligned}
& \left(\ln \left[(\theta / d) \cdot\left(C_{F} / s\right)+1\right] / \theta+\left\lfloor T_{H} /\left(n T_{Q c}\right)\right\rfloor T_{Q c},\right. \\
& \left.\left.\left\lfloor T_{H} /\left(n T_{Q c}\right)\right\rfloor+1\right] T_{Q c}\right)
\end{aligned}
$$

simultaneously, then $t^{\prime}=t^{\prime \prime}$.

\section{Appendix $\mathbf{J}$}

Proof of Proposition 11: Given $n$ and $n_{1}$, minimizing the objective function $T C\left(n_{1}, n, t_{F}\right)$ in (32) with respect to $t_{F}$. We get

$$
\begin{aligned}
\frac{\partial^{2} T C\left(n_{1}, n, t_{F}\right)}{\partial t_{F}^{2}}= & \left\{n _ { 1 } d ( h + C _ { d } \theta ) \cdot \left[\left(n-n_{1}\right) \exp \left[\theta \cdot\left(t_{F}+\left\lfloor T_{H} /\left(n T_{Q c}\right)\right] T_{Q c}\right)\right]\right.\right. \\
& +n_{1} \cdot \exp \left[\theta \cdot\left(\left(T_{r}-n_{1} t_{F}\right) /\left(n-n_{1}\right)+\left\lfloor T_{H} /\left(n T_{Q c}\right)\right] T_{Q c}\right)\right] \\
& \left.+n_{1}^{2} s d \cdot \exp \left[\theta \cdot\left(\left(T_{r}-n_{1} t_{F}\right) /\left(n-n_{1}\right)\right)\right]\right\} /\left(n-n_{1}\right) \geq 0 .
\end{aligned}
$$

Therefore $T C\left(n_{1}, n, t_{F}\right)$ is a convex function. Redefine $\partial T C\left(n_{1}, n, t_{F}\right) / \partial t_{F}=R\left(t_{F}\right), R\left(0^{+}\right)=-\infty<0$ and $R(\infty)=\infty>0$. By the intermediate value theorem, there exist a $t_{F}^{\prime} \in(0, \infty)$ such that $\left.\frac{\partial T C\left(n_{1}, n, t_{F}\right)}{\partial t_{F}}\right|_{t_{F^{\prime}}}=0$. Where 


$$
\begin{aligned}
t_{F}^{\prime}= & \operatorname{Arg}_{\left(t_{F}\right)}\left\{\frac{\partial T C\left(n_{1}, n, t_{F}\right)}{\partial t_{F}}=0\right\}=\operatorname{Arg}_{\left(t_{F}\right)}\left\{( d / \theta ) \cdot \left\{\left\{h n _ { 1 } \left\{1+\exp \left[\theta \cdot\left(t_{F}+\left\lfloor T_{H} /\left(n T_{Q c}\right)\right] T_{Q c}\right)\right]\right.\right.\right.\right. \\
& \left.-\exp \left[\theta \cdot\left[\left(T_{r}-n_{1} t_{F}\right) /\left(n-n_{1}\right)+\left[T_{H} /\left(n T_{Q c}\right)\right] T_{Q c}\right]\right\}\right\}-n h+\theta n_{1}\left\{C_{d} \exp \left[\theta \cdot\left(t_{F}+\left\lfloor T_{H} /\left(n T_{Q c}\right)\right] T_{Q c}\right)\right]\right. \\
& \left.-\exp \left[\theta \cdot\left[\left(T_{r}-n_{1} t_{F}\right) /\left(n-n_{1}\right)+\left[T_{H} /\left(n T_{Q c}\right)\right] T_{Q c}\right]-s \cdot \exp \left[\theta \cdot\left[\left(T_{r}-n_{1} t_{F}\right) /\left(n-n_{1}\right)\right]\right\}\right\}=0\right\}
\end{aligned}
$$

Therefore we can find $t_{F}^{\prime} \in(0, \infty)$ that minimizes $\operatorname{TC}\left(n_{1}, n, t_{F}\right)$. 